\title{
Multiparticle Composites in Density-Imbalanced Quantum Fluids
}

\author{
Evgeni Burovski, Giuliano Orso, and Thierry Jolicoeur \\ Laboratoire de Physique Théorique et Modèles statistiques, Université Paris-Sud, 91405 Orsay, France
} (Received 3 April 2009; revised manuscript received 8 September 2009; published 16 November 2009)

\begin{abstract}
We consider two-component one-dimensional quantum gases with a density imbalance. While generically such fluids are two-component Luttinger liquids, we show that if the ratio of the densities is a rational number, $p / q$, and mass asymmetry between components is sufficiently strong, one of the two eigenmodes acquires a gap. The gapped phase corresponds to (algebraic) ordering of $(p+q)$-particle composites. In particular, for attractive mixtures, this implies that the superconducting correlations are destroyed. We illustrate our predictions by numerical simulations of the fermionic Hubbard model with hopping asymmetry.
\end{abstract}

DOI: 10.1103/PhysRevLett.103.215301

Thanks to recent advances in experimental techniques of dealing with cold gases, it is now feasible to engineer onedimensional (1D) quantum fluids by confining atoms in cigar-shaped traps with tight radial confinement [1]. By devising an appropriate optical lattice it is also possible to construct a weakly coupled array of such 1D "tubes," thus allowing one to study the dimensional crossover from 1D to three dimensions. A number of ongoing and planned experiments deal with two-component mixtures atoms of either statistics, i.e., Fermi-Fermi (FF), Bose-Bose (BB), or Bose-Fermi (BF) mixtures [2]. Most of recent theoretical work dealt with equal-density mixtures, where a rich phase diagram containing both gapped and gapless phases was found [3-5]. For mixtures with unequal densities the ground state is generally found to be a two-component Luttinger liquid $[3,6,7]$. For attractive FF mixtures, superconducting correlations dominate, thus making the ground state a 1D analog of the long-elusive Fulde-Ferrell-LarkinOvchinnikov (FFLO) phase, as confirmed both by Bethe ansatz calculations for integrable models [8] and numerical simulations $[9,10]$. The case of unequal mass mixtureswhere integrable microscopic models are not availablehas been studied analytically by means of effective field theory [3,7], and numerically by Monte Carlo [11] and time-evolving block decimation (TEBD) [10] methods. A common result which emerges is that for strong enough mass asymmetry and/or strong enough attraction the system collapses, while for moderate mass asymmetry and nonzero density imbalance the ground state is again a gapless two-component Luttinger liquid with an FFLOtype algebraic order.

In this Letter we study a generic two-component 1D mixture with density imbalance within the harmonic fluid approach ("bosonization"). We reveal a generic mechanism which, for a certain relation between the densities, opens a gap in the excitation spectrum and completely destroys superconducting correlations. We concentrate on the properties of the FF mixtures, but our predictions are ap-
PACS numbers: 67.10.-j, 03.75.Hh, 03.75.Mn, 71.10.Pm

plicable to BF and BB mixtures with minor modifications. Our findings might also be relevant to spin ladder materials with nonequivalent chains in high magnetic fields. We further corroborate our predictions by density-matrix renormalization group (DMRG) simulations [12] of a Hubbard model with hopping asymmetry.

Consider the mixture of two sorts of fermionic atoms, which we label by a pseudospin index $\sigma=\uparrow, \downarrow$. In the bosonization approach we introduce for each species a pair of scalar fields $\phi_{\sigma}(x)$ and $\theta_{\sigma}(x)$ which vary slowly on the scale of $n_{\sigma}^{-1}$, where $n_{\sigma}$ are the average densities [13]. Using the Haldane construction we write for the field operators $\Psi_{\sigma}^{\dagger}(x) \sim\left(n_{\sigma}-\partial_{x} \phi_{\sigma} / \pi\right)^{1 / 2} \sum_{s} e^{i s\left(\pi n_{\sigma} x-\phi_{\sigma}\right)} e^{-i \theta_{\sigma}}$ where the summation over $s$ runs over odd integers $s$ [14]. For the density operator, $\hat{n}_{\sigma}$, this leads to

$$
\hat{n}_{\sigma}(x) \sim\left(n_{\sigma}-\partial_{x} \phi_{\sigma} / \pi\right) \sum_{s} e^{2 i s\left(\pi n_{\sigma} x-\phi_{\sigma}\right)} .
$$

One of the advantages of the Haldane representation (1) is that an effective low-energy Hamiltonian can be written solely in terms of $\phi_{\sigma}$ and $\Pi_{\sigma}$ [13]. In the noninteracting case it is given by $\mathcal{H}_{\text {free }}=\mathcal{H}_{0}\left(\phi_{\uparrow}\right)+\mathcal{H}_{0}\left(\phi_{\downarrow}\right)$, where

$$
\mathcal{H}_{0}\left(\phi_{\sigma}\right)=\frac{v_{\sigma}}{2 \pi} \int d x\left[K_{\sigma}\left(\pi \Pi_{\sigma}\right)^{2}+K_{\sigma}^{-1}\left(\partial_{x} \phi_{\sigma}\right)^{2}\right],
$$

where $v_{\sigma}$ are Fermi velocities and $K_{\sigma}=1$ the so-called Luttinger parameters equal to one in the free case. In the presence of density-density interactions, $\int d x d x^{\prime} U_{\sigma \sigma^{\prime}}(x-$ $\left.x^{\prime}\right) \hat{n}_{\sigma}(x) \hat{n}_{\sigma}^{\prime}\left(x^{\prime}\right)$, Eq. (2) is modified in several ways. First of all, the $s=0$ terms of Eq. (1) give rise to an acoustic coupling

$$
\mathcal{H}_{1}=g \int d x\left(\partial_{x} \phi_{\uparrow}\right)\left(\partial_{x} \phi_{\downarrow}\right),
$$

where $g$ is a forward scattering constant for the interspin interactions. More importantly, higher harmonics of Eq. (1) generate the terms of the form 


$$
\mathcal{H}_{h}=\sum_{s, s^{\prime}>0} G_{s s^{\prime}} \int d x \cos \left[2\left(s k_{F}^{\uparrow}-s^{\prime} k_{F}^{\downarrow}\right) x-2\left(s \phi_{\uparrow}-s^{\prime} \phi_{\downarrow}\right)\right]+\sum_{s, s^{\prime}>0} \tilde{G}_{s s^{\prime}} \int d x \cos \left[2\left(s k_{F}^{\uparrow}+s^{\prime} k_{F}^{\downarrow}\right) x-2\left(s \phi_{\uparrow}+s^{\prime} \phi_{\downarrow}\right)\right] .
$$

Here $G_{s, s^{\prime}}$ and $\tilde{G}_{s, s^{\prime}}$ are (nonuniversal) amplitudes, and $k_{F}^{\sigma}=\pi n_{\sigma}$ are Fermi momenta. Since the separation of fast and slow variables is inherent in the bosonization treatment, one has to discard in (4) the terms which oscillate on the length scale $\sim k_{F}^{-1}$. Strictly speaking, Eq. (3) is only perturbative in $g$. On the contrary, Eq. (2) is assumed to retain its functional form even in the presence of generic same-spin density-density interaction, with both velocities and Luttinger liquid parameters renormalized by interaction terms beyond Eq. (3) and various irrelevant operators, e.g., band curvature [13]. On a phenomenological level, we can assume Eq. (2) (where, in general, $K_{\sigma} \neq 1$ ) as coming from an underlying microscopic model, with Eqs. (3) and (4) regarded as perturbations.

Equation (4) suggests considering generalized commensurabilities of the form

$$
p n_{\uparrow}-q n_{\downarrow}=0,
$$

where $p$ and $q$ are relatively prime integers. Notice that this condition does not imply the presence of a lattice: we only require the densities to be commensurate with each other. Equation (5) selects from Eq. (4) the terms with $s / s^{\prime}=$ $p / q$, and the Hamiltonian (4) reduces to

$$
\mathcal{H}_{2}=G \int d x \cos 2\left(p \phi_{\uparrow}(x)-q \phi_{\downarrow}(x)\right)
$$

where we only keep the lowest order term, since the scaling dimension of the operator $\cos s \phi$ is $s^{2}$ [15].

We now assume that the densities are commensurate via (5), and analyze the model $\mathcal{H}=\mathcal{H}_{0}\left(\phi_{\uparrow}\right)+\mathcal{H}_{0}\left(\phi_{\uparrow}\right)+$ $\mathcal{H}_{1}+\mathcal{H}_{2}$ defined by (2), (3), and (6). Since in general this model is not exactly solvable, the nature of the phases can, in principle, be determined by an approximate renormalization group (RG) procedure. Rescaling the fields via $\tilde{\phi}_{\uparrow}=p \phi_{\uparrow}$ and $\tilde{\phi}_{\downarrow}=q \phi_{\downarrow}$ the model is brought to the form considered in Ref. [5], where an RG procedure has been carried out including the renormalization of velocities $v_{\sigma}$ [see also Ref. [16] in the fermionic language]. For large velocity asymmetry and strong attractive (repulsive) interactions the system was always found to collapse (phase separate). Barring such an instability, two regimes were found, corresponding to the cosine operator (6) being relevant or irrelevant in the RG sense.

In the regime where the cosine operator Eq. (6) is irrelevant we are left with a bilinear Hamiltonian (2) and (3), which is diagonalized by appropriate linear combinations of the fields [17]. As a result one obtains an effective theory $\mathcal{H}_{\mathcal{A}}$ which features two decoupled massless fields $\varphi_{1,2}$ with corresponding velocities $v_{1,2}$ and Luttinger parameters $K_{1,2}: \mathcal{H}_{\mathcal{A}}=\mathcal{H}_{0}\left(\varphi_{1}\right)+\mathcal{H}_{0}\left(\varphi_{2}\right)$. For an attractive FF mixture, such a theory describes a $1 \mathrm{D}$ analog of the FFLO phase: all correlations are algebraic in real space and the pair correlation function oscillates with the FFLO momentum $Q_{\mathrm{FFLO}}=\left|k_{F}^{\uparrow}-k_{F}^{\downarrow}\right|$.
Another regime corresponds to the case where the cosine in Eq. (6) is relevant in the RG sense. Then the system has a massive mode $\phi_{a}$ and a massless mode $\phi_{b}$. The effective theory $\mathcal{H}_{\mathcal{B}}$ can be written as

$$
\mathcal{H}_{\mathcal{B}}=\mathcal{H}_{\mathrm{sG}}\left(\phi_{a}\right)+\mathcal{H}_{0}\left(\phi_{b}\right),
$$

where $\mathcal{H}_{\mathrm{sG}}\left(\phi_{a}\right)=\mathcal{H}_{0}\left(\phi_{a}\right)+G \int d x \cos 2 \sqrt{2} \phi_{a}$ is the sine-Gordon model for the field $\phi_{a}$. Equation (7) is characterized by two mode velocities $v_{a, b}$ and Luttinger exponents $K_{a, b}$ with $K_{a} \leqslant 1$, so that $\phi_{a}$ is pinned at a minimum of the cosine operator in (7). Closed-form expressions for the parameters of Eq. (7) can be obtained in several limiting cases. Indeed, for $v_{\uparrow}=v_{\downarrow}$, the exact eigenmodes of $\mathcal{H}=\mathcal{H}_{0}\left(\phi_{\uparrow}\right)+\mathcal{H}_{0}\left(\phi_{\downarrow}\right)+\mathcal{H}_{2}$ are

$$
\begin{aligned}
& \phi_{a}=\left(p \phi_{\uparrow}-q \phi_{\downarrow}\right) / \sqrt{2}, \\
& \phi_{b}=\left(q K_{\downarrow} \phi_{\uparrow}+p K_{\uparrow} \phi_{\downarrow}\right) / \sqrt{2},
\end{aligned}
$$

with the Luttinger exponents

$$
K_{a}=\left(p^{2} K_{\uparrow}+q^{2} K_{\downarrow}\right) / 2, \quad K_{b}=K_{a} K_{\uparrow} K_{\downarrow} .
$$

Notice that for higher-order commensurabilities (larger $p$ and $q$ ) smaller values of $K$ are required for $\phi_{a}$ to acquire a gap, cf. Eq. (9).

Deep in the massive phase one can make a crude approximation to the cosine operator in $\mathcal{H}_{2}$ by replacing it with a mass term $\propto\left(p \phi_{\uparrow}-q \phi_{\downarrow}\right)^{2}$. This leads to

$$
\begin{aligned}
v_{b}^{2} & =v_{\uparrow} v_{\downarrow} \frac{\left(p^{2} K_{\uparrow} v_{\downarrow}+q^{2} K_{\downarrow} v_{\uparrow}\right)}{\left(p^{2} K_{\uparrow} v_{\uparrow}+q^{2} K_{\downarrow} v_{\downarrow}\right)}, \\
K_{b} & =\frac{1}{2} K_{\uparrow} K_{\downarrow} \sqrt{v_{\uparrow} v_{\downarrow}} \frac{\left(p^{2} K_{\uparrow}+q^{2} K_{\downarrow}\right)^{2}}{p^{2} K_{\uparrow} v_{\downarrow}+q^{2} K_{\downarrow} v_{\uparrow}},
\end{aligned}
$$

which reduces to (9) for $v_{\uparrow}=v_{\downarrow}$.

We now turn our attention to an interpretation of the theory (7), focusing on the novel regime with $p>1$. Obviously, excitations corresponding to eigenmodes $\phi_{a, b}$ carry both spin and charge. Furthermore, these excitations correspond to multiparticle states in terms of the original $\uparrow, \downarrow$ particles since a particle of the species $\sigma$ corresponds to a $\pi$ kink of the field $\phi_{\sigma}$ [13]. To gain further insight to the structure of the massive phase we consider its correlation properties. We classify operators $\mathcal{O}(x)$ according to whether the asymptotic decay of the correlation functions $\left\langle\mathcal{O}(0) \mathcal{O}^{\dagger}(x)\right\rangle$ for $x \rightarrow \infty$ is exponential, $\propto e^{-\lambda_{\mathcal{O}}|x|}$, or algebraic, $\propto|x|^{-2 \alpha_{O}}$. For equal densities, $p=q=1$, the dominant algebraic order (i.e., the smallest decay exponent $\alpha_{\mathcal{O}}$ ) is found among the two-point operators: the superconducting fluctuations, $\mathcal{O}_{S}=\Psi_{\uparrow} \Psi_{\downarrow}$, and charge density wave, $\mathcal{O}_{\mathrm{CDW}}=\sum_{\sigma, \sigma^{\prime}} \psi_{R \sigma}^{\dagger} \delta_{\sigma \sigma^{\prime}} \psi_{L \sigma^{\prime}}$, and spin density wave, $\mathcal{O}_{\mathrm{SDW}}^{\alpha}=\sum_{\sigma, \sigma^{\prime}} \sigma \psi_{R \sigma}^{\dagger} \sigma_{\sigma \sigma^{\prime}}^{\alpha} \psi_{L \sigma^{\prime}}$. Here $\psi_{L, R \sigma}$ are left- and right-moving fermions, respectively, and $\sigma^{\alpha}$ are the Pauli matrices. 
The case $p \neq q$ is markedly different: First of all, the superconducting correlations described by $\mathcal{O}_{S}$ always decay exponentially, and so do the $x$ and $y$ components of $\mathcal{O}_{\mathrm{SDW}}^{\alpha}$. For the $\mathrm{CDW}$ and $\mathrm{SDW}^{z}$ operators we write $\mathcal{O}_{\mathrm{CDW}}=\mathcal{O}_{\mathrm{LR}}^{\uparrow}+\mathcal{O}_{\mathrm{LR}}^{\downarrow}$ and $\mathcal{O}_{\mathrm{SDW}}^{z}=i\left(\mathcal{O}_{\mathrm{LR}}^{\uparrow}-\mathcal{O}_{\mathrm{LR}}^{\downarrow}\right)$, where the auxiliary operators $\mathcal{O}_{\mathrm{LR}}^{\sigma}=\psi_{L \sigma}^{\dagger} \psi_{R \sigma}$. Using (8) we find for $x \rightarrow \infty$

$$
\left\langle\mathcal{O}_{\mathrm{LR}}^{\uparrow}(0) \mathcal{O}_{\mathrm{LR}}^{\uparrow}(x)^{\dagger}\right\rangle \sim\left|A\left(b_{\uparrow}\right)\right|^{2} e^{-2 i k_{F}^{\dagger} x}|x|^{-2 \alpha_{\uparrow}},
$$

where $\quad \alpha_{\uparrow}=q^{2} K_{b} / 2 K_{a}^{2}, \quad b_{\uparrow}=p K_{\uparrow} / K_{a}, \quad$ and $\quad A\left(b_{\uparrow}\right)=$ $\mid\left\langle\left. e^{\left.i \sqrt{2} \phi_{a} b_{\uparrow}\right\rangle}\right|^{2}\right.$. Likewise, for the $\downarrow$ species the exponent is $\alpha_{\downarrow}=p^{2} K_{b} / 2 K_{a}^{2}$ and the amplitude is $A\left(b_{\downarrow}\right)$ with $b_{\downarrow}=$ $q K_{\downarrow} / K_{a}$. The amplitudes $A(b)$ depend exponentially on $b$ : $\log A(b) \propto 1 / b^{4}$ [18]. We thus see that correlations of $\mathcal{O}_{\text {CDW }}$ and $\mathcal{O}_{\text {SDW }}^{z}$ are both given by a superposition of two power-laws (11) with exponents $\alpha_{\uparrow, L}$-where the slower the decay, the smaller (exponentially smaller) is the corresponding amplitude.

Given the massive mode in the form (8) with $p \neq q$, we construct a compound operator $\mathcal{O}_{p+q}=\Psi_{\downarrow}^{p} \Psi_{\uparrow}^{q}$ which has algebraically decaying correlations. Specializing for the lowest order commensurability (5) with $p=2$ and $q=$ 1 , this corresponds to a "trimer" operator $\mathcal{O}_{2+1}=$ $\Psi_{\downarrow}^{\dagger} \Psi_{\downarrow}^{\dagger} \Psi_{\uparrow}^{\dagger}$. For fermionic $\uparrow$ component the corresponding decay exponent $\alpha_{2+1}=\left(K_{b} / 2 K_{a}^{2}+2 K_{a}^{2} / K_{b}\right) / 2$. We thus see that in this particular case the dominant correlations in the massive phase are the $2 k_{F}$ density waves (11) for $\alpha_{\uparrow}<$ $1 / \sqrt{3}$, and the "trimer" correlations $\mathcal{O}_{p+q}$ for $\alpha_{\uparrow}>1 / \sqrt{3}$. We stress that the competition between $\mathcal{O}_{\mathrm{LR}}$ and $\mathcal{O}_{p+q}$ is generic, in a sense that it holds irrespective of the statistics of $\uparrow$ and $\downarrow$ particles both on the lattice and in the continuum.

Microscopics.-We now focus on the following question: Is there a microscopic model whose low-energy effective theory would be given by Eqs. (5) and (7)?

We start from constructing such a model explicitly in the weak-coupling regime with respect to the interspecies interaction. Namely, for the $\downarrow$ component we take noninteracting fermions (or, equivalently, Tonks bosons) of the mass $m_{\downarrow}$ and (linear) density $n_{\downarrow}$, so that $K_{\downarrow}=1$ and $v_{\downarrow}=\pi n_{\downarrow} / m_{\downarrow}$. For the $\uparrow$ species we take a dipolar Bose gas which is known to be a Luttinger liquid with $K_{\uparrow} \rightarrow$ $\pi\left[6 \zeta(3) n_{\uparrow} r_{0}\right]^{-1 / 2}$ as $n_{\uparrow} r_{0} \rightarrow \infty$ [19]. Here $r_{0}=m_{\uparrow} d^{2} / 2 \pi$ is the effective Bohr radius associated with the dipole moment $d$ and $\zeta$ is the Riemann zeta function. We thus see that for $n_{\uparrow} r_{0}=p^{4} \pi^{2} / 6 \zeta(3)$ we have $K_{\uparrow}=1 / p^{2}$. Furthermore, Galilean invariance fixes the product $v_{\uparrow} K_{\uparrow}=$ $\pi n_{\uparrow} / m_{\uparrow}$ [14]. Constraining the densities via (5) with $p>$ $q=1$, and assuming $m_{\uparrow}=p m_{\downarrow}$ we have both $v_{\uparrow}=v_{\downarrow}$ and $p^{2} K_{\uparrow}=q^{2} K_{\downarrow}=1$ by construction. Now, coupling the $\uparrow$ and $\downarrow$ species via, e.g., a short-range interaction $U \int d x n_{\uparrow}(x) n_{\downarrow}(x)$ with infinitesimal $U$ generates the terms of the form (3) and (6) with $g=U / \pi^{2}$. The eigenmodes of the system are then given by Eqs. (8) and a direct calculation yields $K_{a}=p^{2} K_{\uparrow}\left(1+g \frac{K_{\uparrow}}{v_{\uparrow}} \frac{p}{4 q}\right)+O\left(g^{2}\right)$ and $K_{b}=$ $p^{2} K_{\uparrow}^{2} K_{\downarrow}\left(1-g \frac{K_{\uparrow}}{v_{\uparrow}} \frac{p}{4 q}\right)+O\left(g^{2}\right)$. Thus, having $U<0$ yields $K_{a}<1$ and hence drives the system to the gapped phase, where the gap $\Delta$ is exponentially small: $\ln \Delta \sim-$ const $/ U$. A similar construction can easily be effected for an FF mixture on a lattice. In this case we take for the $\uparrow$ component, e.g., a model with finite-range interactions [20].

In the example above we engineer the theory (5) and (7) by coupling a majority of light and noninteracting $\downarrow$ species to the minority of heavy particles $\uparrow$, which have strong repulsions among themselves. Such a construction is somewhat $a d h o c$, and requires fine-tuning. A much more natural alternative is provided by a simple observation: even purely local interspecies coupling $U$ generates long-range effective interactions in higher orders of perturbation theory. Thus, having finite $U$ and $m_{\uparrow} \neq m_{\downarrow}$ should be sufficient to divert the RG flow towards the theory (7). In this case we expect a light minority component to provide an effective coupling between heavy particles of majority species.

To this end we consider an asymmetric attractive $(U<$ 0) Hubbard model

$$
H_{\mathrm{aH}}=-\sum_{\langle i j\rangle \sigma} t_{\sigma}\left(c_{i, \sigma}^{\dagger} c_{j, \sigma}+\text { H.c. }\right)+U \sum_{i} \hat{n}_{i \uparrow} \hat{n}_{i \downarrow},
$$

where $c_{i \sigma}$ annihilates a fermion with spin $\sigma$ on a site $i \in$ $[1, L]$ of a chain lattice of length $L,\langle i j\rangle$ stands for pairs of nearest neighbor sites, $\hat{n}_{i \sigma}=c_{i \sigma}^{\dagger} c_{i \sigma}$, and $t_{\sigma}$ are hopping amplitudes for the spin-up and spin-down components. For $\eta \equiv t_{\downarrow} / t_{\uparrow}=1$ the model (12) is solvable by Bethe ansatz techniques even in the presence of density imbalance. For $n_{\uparrow} \neq n_{\downarrow}$ the ground state is of the FFLO type [8], which, in present language, corresponds to a gapless fixed point theory $\mathcal{H}_{\mathcal{A}}$-see [21] for a detailed discussion.

For $t_{\uparrow} \neq t_{\downarrow}$, the model (12) is no longer integrable, and we resort to numerical simulations using DMRG technique [12]. We use lattices of up to $L=80$ sites with open boundary conditions and DMRG truncation of up to $N_{s}=$ 400 states. We calculate single-particle density matrices $\rho_{\sigma}(x)=\left\langle c_{L / 2, \sigma} c_{L / 2+x, \sigma}^{\dagger}\right\rangle$ and pair-pair correlations $\Gamma(x)=$ $\left\langle\mathcal{P}_{L / 2} \mathcal{P}_{L / 2+x}^{\dagger}\right\rangle$, where $\mathcal{P}_{j}=c_{j \uparrow} c_{j \downarrow}$ is the lattice version of the superconducting operator $\mathcal{O}_{S}$ and $\langle\cdots\rangle$ denotes an expectation value over the ground state.

Figure 1 shows typical results for the pair-pair correlations $\Gamma(x)$. We find that for small enough hopping asymmetry, $\eta>\eta_{c 1}$, the long-distance decay of both singleparticle (not shown) and two-particle correlations is consistent with the FFLO-type laws $\Gamma(x) \propto \cos \left(Q_{\mathrm{FFLO}} x\right)|x|^{-\gamma}$ and $\rho_{\sigma}(x) \propto \cos \left(k_{F}^{\sigma} x\right)|x|^{-\beta}$. On the contrary, once the hopping asymmetry exceeds some critical value and the densities are commensurate via (5), the power-law decays change to exponentials, namely $\Gamma(x) \propto e^{-|x| \lambda} \times$ $\cos \left(Q_{\mathrm{FFLO}} x\right)|x|^{-\gamma^{\prime}}$ and likewise for $\rho_{\sigma}(x)$, thus unequivocally signaling the presence of a gap. Violating the relation (5) destroys the gap, and the correlation functions decay algebraically again. 


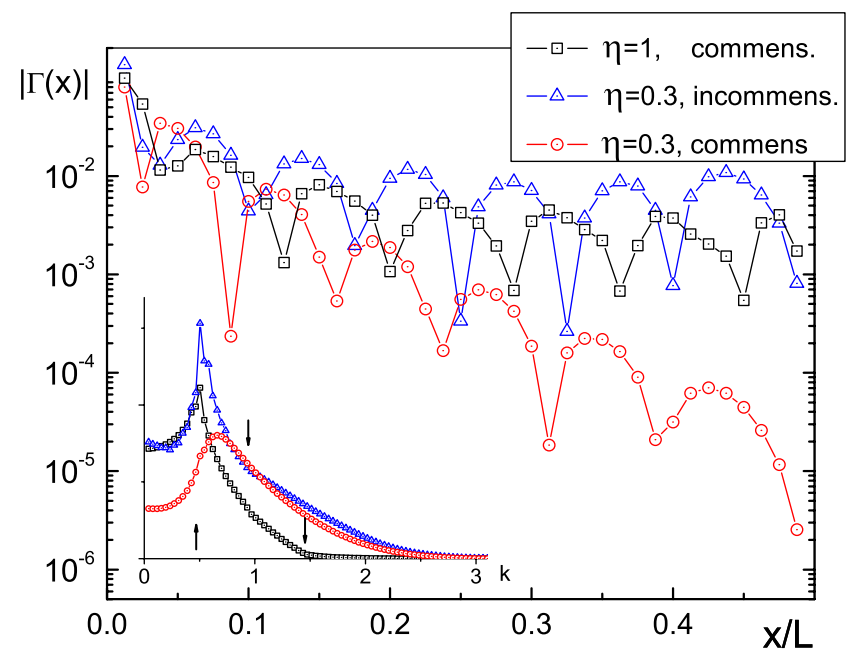

FIG. 1 (color online). Superconducting correlation function $\Gamma(x)$ for the asymmetric Hubbard model (12) for $n_{\downarrow}=2 n_{\uparrow}=$ 3/10: $\eta=1$ (black squares) and $\eta=0.3$ (red circles). Shown by blue triangles is $\Gamma(x)$ for $\eta=0.3$ and "incommensurate" densities $n_{\uparrow}=17 / 80$ and $n_{\downarrow}=29 / 80$. Hubbard coupling is $U=$ $-5 t_{\uparrow}$ and the system size $L=80$. Lines are guides to the eye. Inset: Fourier transform of $\Gamma(x)$, same color coding. Arrows indicate the characteristic momenta: $k_{F}^{\downarrow}-k_{F}^{\uparrow}, k_{F}^{\downarrow}$ and $k_{F}^{\uparrow}+k_{F}^{\downarrow}$, respectively, for the "commensurate" densities $n_{\downarrow}=2 n_{\uparrow}=$ $3 / 10$. We stress that in all these simulations the density distributions for both components are uniform apart from Friedel oscillations induced by the open boundary conditions.

The inset in Fig. 1 shows the superconducting correlation function in momentum space. Compared to the Hubbard limit, we see that the mass imbalance leads to an overall broadening of the distribution, which now extends well beyond $k_{F}^{\uparrow}+k_{F}^{\downarrow}$. In addition, the opening of the gap at commensurate filling depletes the superconducting correlation at small momentum. Detailed investigation of the asymmetric Hubbard model (12) is beyond the scope of this Letter and will be reported elsewhere [22].

Conclusions and outlook.-Summarizing, we have revealed a generic mechanism of opening a gap in twocomponent quantum fluids with density imbalance in one spatial dimension. The gapped phase appears once interactions and mass asymmetry between components is strong enough, and the densities satisfy Eq. (5). Depending on the microscopic details, the system develops quasi-long-range ordering of either $2 k_{F}$ density waves or of peculiar $(p+q)$-particle composites. The proposed mechanism applies to mixtures of particles of either statistics, and does not require the presence of a lattice. Experimental signatures of the proposed state include (i) the disappearance of the superconducting ordering, and (ii) appearance of the $(p+q)$-particle composites, which can be detected, e.g., by noise correlation measurements in the time-of-flight absorption imaging using the techniques discussed in Ref. [23].

We are indebted to T. Vekua for illuminating discussions. This work was supported in part by Institut
Francilien de Recherche sur les Atomes Froids (IFRAF) and ANR under Grant No. 08-BLAN-0165-01. G. O. was also supported by the Marie Curie Fellowship under Contract No. EDUG-038970. Numerical simulations were performed using DMRG application of the ALPS libraries [24].

[1] I. Bouchoule, N. J. Van Druten, and C.I. Westbrook, arXiv:0901.3303; H. Moritz, Th. Stöferle, K. Günter, M. Köhl, and T. Esslinger, Phys. Rev. Lett. 94, 210401 (2005); S. Aubin et al., J. Low Temp. Phys. 140, 377 (2005).

[2] For a review of both theoretical and experimental status see, e.g., S. Giorgini, L. P. Pitaevskii, and S. Stringari, Rev. Mod. Phys. 80, 1215 (2008), and references therein.

[3] M. A. Cazalilla and A. F. Ho, Phys. Rev. Lett. 91, 150403 (2003).

[4] M. A. Cazalilla, A. F. Ho, and T. Giamarchi, Phys. Rev. Lett. 95, 226402 (2005).

[5] L. Mathey, Phys. Rev. B 75, 144510 (2007).

[6] L. Mathey and D.-W. Wang, Phys. Rev. A 75, 013612 (2007).

[7] W.-L. Lu, Z.-G. Wang, S.-J. Gu, and H.-Q. Lin, arXiv:0902.1021.

[8] G. Orso, Phys. Rev. Lett. 98, 070402 (2007); H. Hu, X.-J. Liu, and P. D. Drummond, Phys. Rev. Lett. 98, 070403 (2007).

[9] A. E. Feiguin and F. Heidrich-Meisner, Phys. Rev. B 76, 220508(R) (2007); M. Rizzi et al., Phys. Rev. B 77, 245105 (2008); G. G. Batrouni et al., Phys. Rev. Lett. 100, 116405 (2008); M. Tezuka and M. Ueda, Phys. Rev. Lett. 100, 110403 (2008).

[10] B. Wang, Han-Dong Chen, and S. Das Sarma, Phys. Rev. A 79, 051604(R) (2009).

[11] G. G. Batrouni et al., Europhys. Lett. 86, 47006 (2009).

[12] S. R. White, Phys. Rev. Lett. 69, 2863 (1992); U. Schollwöck, Rev. Mod. Phys. 77, 259 (2005).

[13] See, e.g., Th. Giamarchi, Quantum Physics in One Dimension (Clarendon Press, Oxford, 2004), and references therein.

[14] F. D. M. Haldane, Phys. Rev. Lett. 47, 1840 (1981).

[15] For lattice models there is an additional possibility that $p n_{\uparrow}+q n_{\downarrow}=$ integer $/ a_{0}$, where $a_{0}$ is the lattice constant. In this case an additional term of the form $\cos \left(p \phi_{\uparrow}+q \phi_{\downarrow}\right)$ would appear in the Hamiltonian. The effects due to such term are similar to those due to Eq. (8).

[16] K. Penc and J. Sólyom, Phys. Rev. B 41, 704 (1990).

[17] T. Kimura, K. Kuroki, and H. Aoki, Phys. Rev. B 53, 9572 (1996).

[18] S. Lukyanov and A. Zamolodchikov, Nucl. Phys. B493, 571 (1997); arXiv:hep-th/9611238.

[19] R. Citro et al., Phys. Rev. A 75, 051602(R) (2007).

[20] G. Gómez-Santos, Phys. Rev. Lett. 70, 3780 (1993).

[21] E. Zhao and W. V. Liu, Phys. Rev. A 78, 063605 (2008).

[22] G. Orso, E. Burovski, and Th. Jolicoeur, arXiv:0907.1533.

[23] A. Kuklov and H. Moritz, Phys. Rev. A 75, 013616 (2007).

[24] A. F. Albuquerque et al. (ALPS Collaboration), J. Magn. Magn. Mater. 310, 1187 (2007). 